\title{
miRandola: extracellular circulating microRNAs database
}

\author{
F. Russo', S. Di Bella', G. Nigita², V. Macca², A. Laganà ${ }^{3}$, R. Giugno', A. Pulvirenti', A. Ferro' $\bowtie$ \\ 'Department of Clinical and Molecular Biomedicine, University of Catania, Catania, Italy \\ ${ }^{2}$ Department of Mathematics and Computer Science, University of Catania, Catania, Italy \\ ${ }^{3}$ Department of Molecular Virology, Immunology and Human Genetics, Comprehensive Cancer Center, The Ohio State \\ University, Columbus $\mathrm{OH}$, United States
}

\section{Motivations}

MicroRNAs (miRNAs) are small (approximately 22 nt) noncoding RNAs that play an important role in the regulation of various biological processes through their interaction with cellular messenger RNAs. They are frequently dysregulated in cancer and have shown promise as tissue-based markers for cancer classification and prognostication. Extracellular miRNAs in serum, plasma, saliva, urine and other body fluids have recently been shown to be associated with various pathological conditions including cancer. miRNAs circulate in the bloodstream in a highly stable, extracellular form, thus they may be used as blood-based biomarkers for cancer and other diseases. Circulating miRNAs are protected by encapsulation in membrane-bound vesicles such as exosomes, but the majority of circulating miRNAs in human plasma and serum cofractionate with Argonaute2 (Ago2) protein, rather than with vesicles. In the present work, we performed a comprehensive classification of different extracellular circulating miRNA types. A direct link to the knowledge base miRò together with the inclusion of datamining facilities allow users to infer possible biological functions of the circulating miRNAs and their connection with the phenotype. To our knowledge miRandola is the first database that provides information about all kind of extracellular miRNAs and we believe that it will constitute a very important resource for researchers.

\section{Methods}

miRandola is a manually curated database of circulating miRNAs. The database catalogs infor- mation from both published (from literature and public resources) and unpublished studies (from direct researchers submission). The database include information about miRNAs and their extracellular form, samples, fluids and data sources. It has been created using MySQL, Apache and PHP. For better retrieval and analysis of the miRNA data we have integrated various tools such as search, advanced search and browsing. The search results provide links to miR, our miRNA knowledge base to help users to find useful information about miRNAs and their targets. Finally, an export function allows the download of the search results in various formats (CSV, XLS, TXT).

\section{Results}

miRandola contains around 1700 entries. miRNAs are classified in three categories, based on their extracellular form: miRNA-Ago2, miRNA-exosome and miRNA-circulating. The latter is used when authors don't distinguish between Ago2 and exosome and constitutes the largest group. Simple detection and amplification methods, tissue-restricted expression profiles, and sequence conservation between human and model organisms make extracellular miRNAs ideal candidates for noninvasive biomarkers for the diagnosis and the study of various physiopathological conditions. A comprehensive classification of different extracellular circulating miRNA types is needed to help researchers to find miRNA signatures in human cancer and other diseases.

\section{Availability \\ http://ferrolab.dmi.unict.it/}

